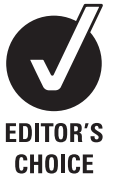
School of Medicine, Cardiff University, Cardiff, UK ${ }^{2}$ Childhood Cancer Research Unit, Astrid Lindgren's Children's Hospital, Karolinska Institutet, Stockholm, Sweden

\section{Correspondence to}

Professor G M Vujanić, Department of Histopathology, School of Medicine, Cardiff University, Heath Park, Cardiff CF14 4XN, UK; vujanic@cf. ac.uk

Accepted 3 August 2009 Published Online First 16 August 2009

\title{
The pathology of Wilms' tumour (nephroblastoma): the International Society of Paediatric Oncology approach
}

\author{
G M Vujanić, ${ }^{1}$ B Sandstedt ${ }^{2}$
}

\section{ABSTRACT}

In the International Society of Paediatric Oncology renal tumour trials, preoperative chemotherapy has been successfully applied with resulting reduction of tumour rupture and increased favourable stage distribution of nephroblastoma. Postoperative treatment includes chemotherapy and sometimes radiotherapy in a riskadapted approach based on histological sub-classification and stage of the tumour. However, preoperative chemotherapy alters the tumour's histological features and distribution of subtypes, and makes staging more difficult. The paper highlights the most common practical diagnostic difficulties that a pathologist is faced with in dealing with pretreated nephroblastomas. It emphasises the importance of a systematic, step-by-step analysis based on adequately sampled material, in order to accurately sub-classify a nephroblastoma as a low, intermediate or high risk tumour and assign its genuine stage. Finally, it outlines the standard operating procedure for submission of renal tumours for rapid central pathology review which allows the treating oncologists to apply the optimal treatment protocol.

Renal tumours comprise 7-8\% of all tumours in the first 15 years of life. Wilms' tumour (WT) or nephroblastoma is by far the most common ( $~ 85 \%$ of cases), followed by renal cell carcinomas ( $\sim 3-5 \%)$, mesoblastic nephroma ( 3\%), clear cell sarcoma of the kidney $(\sim 3-4 \%)$, rhabdoid tumour of the kidney $(\sim 2 \%)$ and miscellaneous rare tumours $(\sim 2 \%) .{ }^{1}$ Accurate histological diagnosis and staging of these tumours are critical because their treatment and prognosis are very different. Since they are rare, they still represent a diagnostic and therapeutic challenge, ${ }^{2}$ and it is important to treat and study them through large national and international multicentre collaborative trials which include centralised pathological review in order to verify the diagnosis and stage of cases entered on the trials.

The first multicentre trial started in 1969 in the United States through the National Wilms' Tumour Study group (NWTS; now part of the Children's Oncology Group, COG) with the most important objective being the establishment of optimal treatment for WT. NWTS/COG have been treating WTs with surgery first, followed by, if necessary, postoperative chemotherapy and radiotherapy. ${ }^{3}$ On the other hand, preoperative therapy has been an essential part of the International Society of Paediatric Oncology (SIOP) treatment strategy since its first trials. In the first two trials (SIOP 1, 1971-74; and SIOP 2, 1974-76) preoper- ative radiotherapy was used. Since the third trial (SIOP 5, 1977-79) it has been replaced with preoperative chemotherapy which has shown very similar results in terms of preventing tumour rupture(s) and inducing more favourable stage distribution, with more stage I tumours requiring less postoperative therapy (SIOP 5, 1977-79; SIOP 6, 1980-86; SIOP 9, 1987-93; and SIOP 93 01, 1993-2001). ${ }^{4}$ Due to the different therapeutic approaches between the two groups, there are further differences in histological subclassification and staging (see below), making a direct comparison of their results complicated.

In 1978, Beckwith and Palmer introduced a histological classification of primarily operated WTs with two main groups-anaplastic and nonanaplastic - which became a basis for their treatment in NWTS/COG trials. ${ }^{5}$ By studying the correlation between the histological features and survival of WTs that received preoperative chemotherapy from the early SIOP trials, it became apparent that they could be sub-classified into three treatment groups: favourable, standard and unfavourable histology groups. ${ }^{6}$ In the more recent classifications (SIOP 9301 and the current SIOP 2001), they have been renamed into low risk, intermediate risk and high risk tumours (table 1). ${ }^{78}$ This concept of stratification of tumours into low, intermediate and high risk groups has later been followed in other tumours of childhood, including rhabdomysarcomas, neuroblastomas, hepatoblastomas and germ cell tumours.

Further analyses of WT subtypes from SIOP trials have resulted in removal from or addition of certain subtypes to the risk groups. So, for example, WT with fibroadenoma-like structures, which was earlier regarded as a favourable histology tumour, has disappeared from the new classification since it has been recognised that it represented a pattern of growth of some WTs rather than a distinct histological type. ${ }^{9}$ On the other hand, it has been shown that a tumour's response to preoperative chemotherapy is an indicator of good prognosis, ${ }^{10}$ so completely necrotic WT has been placed in the low risk tumour group (table 1). ${ }^{8}$ In the current SIOP 2001 trial (2001-), other WT types including epithelial, stromal and regressive (sub)type with $>90 \%$ necrosis are being investigated since preliminary results have indicated that their prognosis is better than for other WT types from the intermediate risk group. ${ }^{11} 12$ In the same way, the presence of a certain amount of blastema after preoperative chemotherapy clearly indicates its non- 
Table 1 The revised International Society of Paediatric Oncology (SIOP) working classification of renal tumours of childhood (2001)

\section{Low risk tumours}

- Mesoblastic nephroma

- Cystic partially differentiated nephroblastoma

- Nephroblastoma-completely necrotic (following preoperative chemotherapy)

\section{Intermediate risk tumours}

- Nephroblastoma-epithelial type

- Nephroblastoma-stromal type

- Nephroblastoma-mixed type

- Nephroblastoma-regressive type

- Nephroblastoma-focal anaplasia type

\section{High risk tumours}

- Nephroblastoma-blastemal type

- Nephroblastoma-diffuse anaplasia type

- Clear cell sarcoma of the kidney

- Rhabdoid tumour of the kidney

responsiveness to chemotherapy, and blastemal type WT has been shown to be associated with poorer outcome ${ }^{13}$ and is therefore moved into high risk tumour group. ${ }^{8}$

According to the SIOP WT 2001 trial protocol, renal tumours in children are treated with preoperative chemotherapy consisting of two drugs given over a period of 4 weeks. Unlike in the rest of the SIOP, in the UK a histological diagnosis made on percutaneous cutting needle ("tru-cut") biopsy is required before preoperative chemotherapy. ${ }^{14}{ }^{15}$ Chemotherapy is followed by surgery and further chemotherapy and/or radiotherapy, if necessary, depending on the tumour's histological subtype and stage.

The pathologist has a critical role in the following:

- Making an accurate histological diagnosis.

- Assigning the tumour's histological subtype and risk group.

- Making a precise evaluation of the abdominal stage of the tumour (even in children with stage IV disease, local staging is crucial in determining the use of radiotherapy).

For pathologists, there is one major disadvantage of preoperative chemotherapy-it significantly alters the histological features of WT, resulting in different histological patterns and distribution of subtypes from those treated with immediate surgery. In the earlier SIOP study, in the immediately operated WTs the most common subtype was mixed (45.1\%), followed by blastemal (39.4\%) and epithelial predominant (15.5\%), whereas in tumours that received preoperative chemotherapy, the most common type was regressive (37.6\%), followed by mixed $(29.4 \%)$, stromal (14\%), blastemal (9.3\%) and epithelial predominant (3.1\%); $6.6 \%$ of tumours were completely necrotic. ${ }^{13}$ Preoperative chemotherapy is more likely to destroy blastema and (less differentiated) epithelial elements, while it induces maturation especially in the stromal component, where rhabdomyoblastic differentiation is much more common than in primarily operated tumours. The typical chemotherapy-induced changes of treated WTs are a mixture of coagulative-type necrosis of small round cells or neoplastic tubules consisting of pink, necrotic nuclei, consistent with coagulative necrosis of blastemal cells or neoplastic tubules, fibrosis, hypocellular stroma containing foamy and/or haemosiderin-laden macrophages, and haemorrhage.

In order to subtype a WT, a pathologist has to evaluate it in a particular order, as follows:

- Assess the percentage of necrosis/regressive changes caused by chemotherapy.
- If only necrosis/regressive changes are present, with no viable tumour identified in an adequately sampled tumour (see below), it is regarded as completely necrotic WT.

- If regressive changes comprise more than two-thirds of a tumour mass, it is a regressive type.

- If regressive changes comprise less than two-thirds of a tumour mass, the viable tumour is sub-classified on the basis of the viable components present (blastemal, epithelial, stromal or mixed type).

- If focal anaplasia is found, the tumour should still be subclassified on the basis of other components.

The criteria for subtyping a nephroblastoma in the SIOP WT 2001 classification are detailed elsewhere. ${ }^{8}$ They include new definitions of certain WT types which have been introduced in order to re-emphasise that they differ from WTs that are treated with no preoperative chemotherapy (table 2). Therefore, it is very important not to confuse what is called epithelial or stromal type WT in SIOP WT 2001 classification with epithelial or stromal predominant WT in the NWTS/COG classifications. The SIOP WT 2001 criteria for epithelial or stromal type WT are that the epithelial or stromal component comprises more than $66 \%$ of the viable tumour, but the rest of the tumour may contain only up to $10 \%$ of blastema (if it contains more, than it is regarded as mixed type). On the other hand, in the NWTS/COG classification, the tumour is called epithelial or stromal predominant if the epithelial or stromal component comprises more than $66 \%$ of the tumour, irrespective of the remaining tumour component(s). So, for example, a WT consisting of $70 \%$ epithelial and $30 \%$ blastemal components would be regarded as epithelial predominant WT in NWTS/COG classification, whereas the same tumour would be not be regarded as epithelial but mixed type in the SIOP classification.

\section{COMMON PROBLEMS IN ASSESSMENT OF PRETREATED WILMS' TUMOURS}

In assessing a pretreated WT, a diagnostic pathologist is faced with a number of problems. Some which may significantly influence tumour's sub-classification and, consequently, treatment are as follows:

- Completely necrotic tumour-was it a WT? Even in completely necrotic WTs, one can often see the contours of pre-existing tumour components (figure 1A,B). If not, look for nephrogenic rests which are only associated with WTs. Finally, if none of these is seen/found, it is still reasonable to assume that the tumour was a WT since other renal tumours usually do not respond so well to relatively mild preoperative chemotherapy that is given for WTs.

- Occasional tubules and small foci of stroma in otherwise completely necrotic tumour (figure 1C). These findings are regarded as nephrogenic remnants and should not be taken into account for subtyping purposes, so the tumour should be

Table 2 Histological criteria for Wilms' tumour subtyping in SIOP WT 2001 trial

\begin{tabular}{lclll}
\hline \multirow{2}{*}{ Tumour type } & \multirow{2}{*}{$\begin{array}{c}\text { CIC } \\
(\%)\end{array}$} & \multicolumn{2}{l}{ Histological features (\% of a tumour) } \\
\cline { 4 - 5 } & Epithelium & Stroma & Blastema \\
\hline Completely necrotic & 100 & 0 & 0 & 0 \\
Regressive & $>66$ & $0-33$ & $0-33$ & $0-33$ \\
Mixed & $<66$ & $0-65$ & $0-65$ & $0-65$ \\
Epithelial & $<66$ & $66-100$ & $0-33$ & $0-10$ \\
Stromal & $<66$ & $0-33$ & $66-100$ & $0-10$ \\
Blastemal & $<66$ & $0-33$ & $0-33$ & $66-100$ \\
\hline
\end{tabular}

$\mathrm{CIC}$, chemotherapy-induced changes. 
Figure 1 Completely necrotic Wilms' tumour. (A) Non-viable ("ghost") blastemal cells separated by septa. (B) Non-viable epithelial (tubular) structures clearly identifying a tumour as a Wilms' tumour. (C) A small group of tubules surrounded by non-viable tumour - they are regarded as nephrogenic remnants and should be ignored for tumour's subtyping. (D) "Dying" blastemal cells with still vaguely recognisable nuclear morphology - should be regarded as non-viable tumour and not taken into account for tumour's subtyping. (E) Hypocellular tumour stroma can be indistinguishable from hypocellular areas with chemotherapy-induced changes. (F) Rare rhabdomyoblasts in a hypocellular stroma indicate it is a genuine, viable tumour stroma.
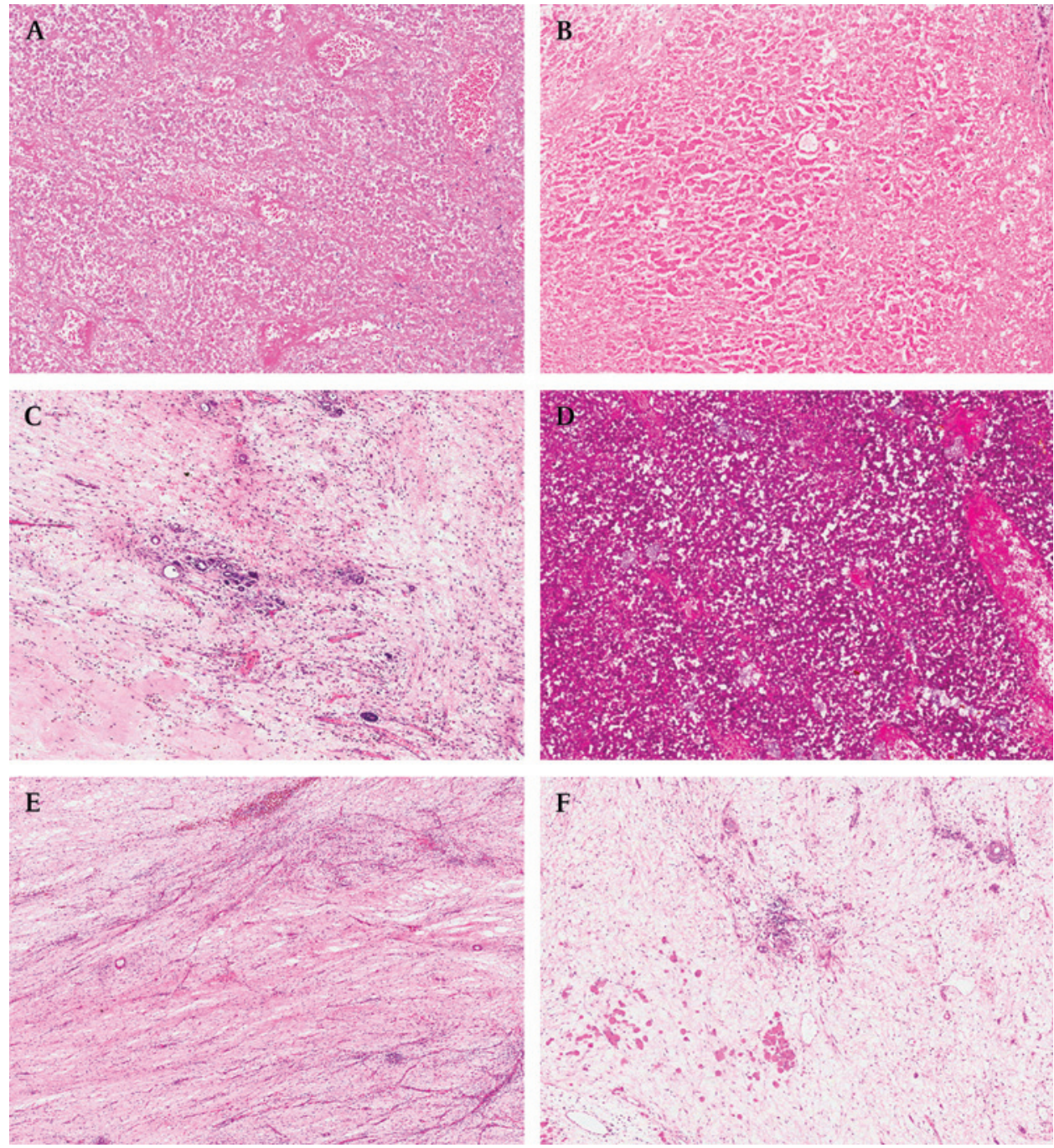

classified as a completely necrotic type (low risk) rather than a regressive type (intermediate risk).

- Necrotic versus viable tumour. There are no clear-cut criteria on the basis of which one could say with absolute certainty when the tumour is not viable any longer. It is relatively easy to recognise genuinely "dead" tumour where either "ghost" tumour cells (figure 1A, B) or foamy macrophages are usually seen, but it is not so easy when tumour cells are "dying" and their nuclear morphology is disappearing but is still vaguely recognisable (figure $1 \mathrm{D}$ ). We regard such areas as non-viable and would not take them into account for subtyping a tumour.

- Hypocellular tumour stroma or chemotherapy-induced changes. It is not always possible to distinguish genuine, hypocellular tumour stroma (figure 1E) from chemotherapy-induced changes, and one should look for other clues such as a finding of rare rhabdomyoblasts (figure 1F) (which would indicate that it is a genuine viable tumour stroma) or foamy macrophages (which would indicate that such areas represent chemotherapy-induced changes).

- More or less than $66 \%$ blastema? (ie, blastemal type (high risk) versus mixed type (intermediate risk) with a lot of blastema). This is one of the most challenging dilemmas because of very serious implications for postoperative treatment and, again, there is no simple answer. The estimation of the percentage of tumour components is semi-quantitative, and by looking at the series of slides from a tumour, a pathologist builds a picture in his/her brain (like a jigsaw) and ends up with an estimate. The histological features of a WT may vary considerably, so sometimes it is necessary to estimate tumour components slide by slide, and add them up. But all these methods are subjective and sometimes one just has to rely on expert opinion (which is, admittedly, equally subjective).

- Anaplasia or not? To make a diagnosis of anaplasia, all three criteria have to be confirmed, including the presence of atypical tri/multipolar mitotic figures, marked nuclear enlargement, with diameters at least three times those of adjacent cells of the same type, and the presence of hyperchromatic tumour cell nuclei (figure 2A). If there are large hyperchromatic nuclei but no atypical mitoses, one should assess the overall mitotic activity. If it is low, it may be very difficult to find atypical mitoses and in such cases the finding of just one atypical mitosis is enough. Curiously, anaplasia still represents the biggest problem in Wilms' tumour histology (in the NWTS 5 series of anaplastic WTs, 39\% cases were misdiagnosed by the institutional pathologists). ${ }^{16}$ Anaplasia may occur in the epithelial, blastemal or even stromal component of WT and it can be either focal or diffuse. ${ }^{17}$ The definition of focal anaplasia underlines the distribution of anaplasia which has been defined as the presence of a clearly defined focus within a primary intrarenal tumour, without evidence of anaplasia or prominent 
Figure 2 (A) Anaplastic Wilms' tumour with all three features including atypical mitoses, marked nuclear enlargement and hyperchromasia. (B) Focal anaplasia surrounded by chemotherapy-induced changes, making it easier to spot. (C, D) Pseudoanaplasia in the skeletal muscle.

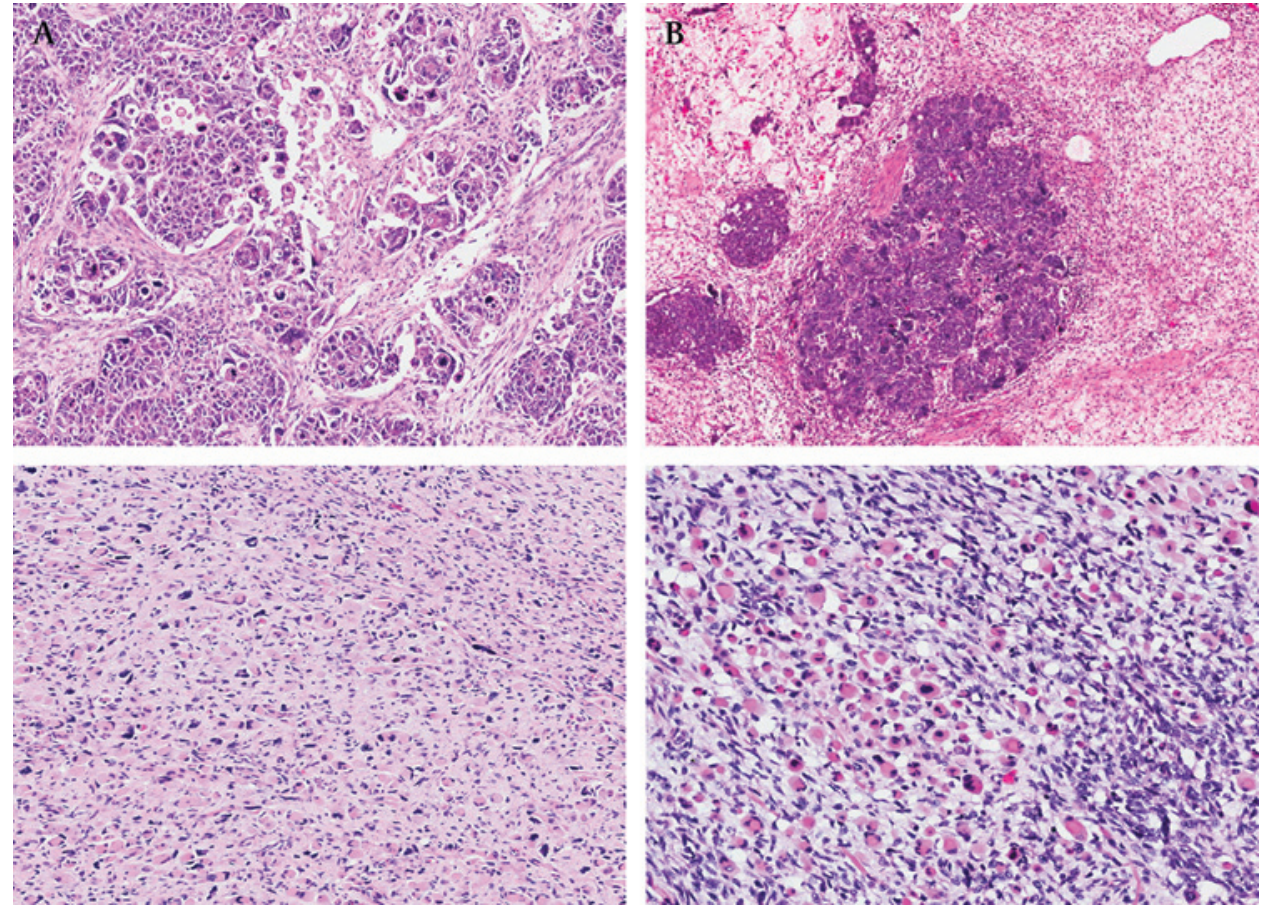

nuclear atypia in other sites (figure 2B). Very rarely, focal anaplasia may have more than one focus, but each of them has to be surrounded on all slides by non-anaplastic tumour. The finding of anaplasia in any other circumstances is regarded as diffuse anaplasia. Preoperative chemotherapy does not eradicate or generate anaplasia (although there are cases of bilateral nonanaplastic WTs treated with prolonged preoperative chemotherapy which eventually became anaplastic; personal experience). There are a number of histological findings which may mimic anaplasia such as fused or smudged masses of nuclear chromatin due to technical artefact, overlapping cells in thick sections, stain precipitate and circulating megakaryocytes. The most frequently observed pseudo-anaplastic changes are seen in the stromal rhabdomyoblasts which may exhibit large, hyperchromatic, multinucleated, bizarre nuclei as a response to chemotherapy (figure 2C,D).

\section{STAGING OF PRETREATED WILMS' TUMOURS}

Another problem with preoperative chemotherapy is that it makes staging more difficult. However, the results from both SIOP and NWTS/COG trials show that staging is a major problem, not only in pretreated but also in primarily operated tumours. ${ }^{2} 16$ One of the reasons is that these tumours are usually large and distort the normal architecture of the kidney and its anatomical structures, such as the renal sinus and capsule.

In order to obtain accurate information about the stage of the tumour, the nephrectomy specimen has to be dealt with as detailed in box 1. The two most important steps are to have a (preferably a photo) block guide (figure 3 ) and to ink the surface of the specimen. Ideally, at least one whole slice of a tumour should be blocked (figure 3), but additional blocks should be taken (box 2)

Table 3 shows the staging criteria followed in the SIOP WT 2001 Trial. It is important to remember that the purpose of giving preoperative chemotherapy is to induce a more favourable stage distribution by down-staging tumours. For that reason, staging is based on objective histological findings at the time of nephrectomy rather than on assumptions of what a pathologist thinks had been there before preoperative chemotherapy was given. For example, if there is a viable tumour outside of the kidney, in the perirenal fat, but it is covered by a pseudocapsule (which is likely to be formed due to preoperative chemotherapy), the tumour is still regarded as stage I (figure 4A).

In the current SIOP WT 2001 trial, chemotherapy-induced changes are not taken into account for staging purposes if present in the perirenal fat or renal sinus (either its soft tissues or vessels) (figure 4). ${ }^{18}$ However, if present at the resection margins in the perirenal fat, in the renal vein or inferior vena cava thrombi, or in lymph nodes (figure 5), they are regarded as a criterion for stage III.

Some staging problems/dilemmas are as follows:

- Viable tumour is present around large blood vessels but no other structures of the renal sinus are obvious. WTs often contain large intra-tumoural vessels and they should not be confused with the renal sinus vessels. The most reliable criterion

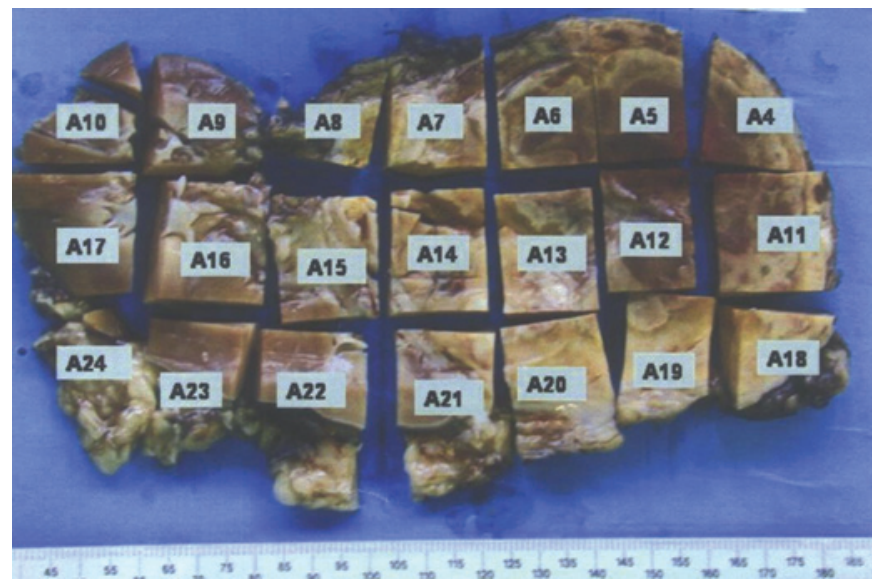

Figure 3 A photo block guide clearly showing where the blocks have been taken from, making accurate staging possible (courtesy of $\mathrm{Dr}$ GK Kokai, Royal Liverpool Children's Hospital Alder Hay, Liverpool, UK). 


\section{Box 1 Handling of the nephrectomy specimen}

- The specimen should be first weighed, measured and photographed. Areas with suspected ruptures and/or invasion should be inked in different colours from the rest of the specimen. A tumour capsule must not be stripped as it would make determination of growth beyond the capsule impossible.

- Perirenal and perihilar lymph nodes should be searched for and sampled separately.

- The renal vein, artery and ureter should be identified and sampled near the resection margin.

- The surface of the whole specimen should be inked and allowed to dry before opening the specimen. This is a crucial step and should always be done; otherwise it might not be possible to stage the tumour.

- The specimen should be opened by a longitudinal incision to reveal the tumour and its relation to the kidney, capsule and renal sinus.

- The cut surface should be photographed and its macroscopic appearance recorded. The tumour dimensions should be measured and the percentage of necrosis assessed.

- Samples of fresh tumour and normal renal tissue should be taken for tumour banking and molecular studies.

- The specimen should be fixed in $4 \%$ buffered formalin for 24-48 hours, according to the usual procedure of the laboratory. Several additional cuts can be made parallel to the initial cut to divide the specimen into "slabs" for better fixation.

- After fixation, blocks should be taken according to the recommended protocol (box 2).

for identifying the renal sinus is the presence of nerves as they are never present in the tumour (figure 4C).

- There is no clearly defined (pseudo) capsule and fibrosis is present at the resection margin(s). This should not be regarded as
Box 2 Blocks selection from nephrectomy for renal tumours

After fixation, at least one slice of the tumour should be sampled and carefully recorded (figure 3 ).

In addition, the following blocks should be taken and recorded:

- Areas of doubtful resection, as marked by the surgeon or pathologist.

- Sinus lymph nodes when present.

- Other lymph nodes.

- Renal sinus, ureter and sinus vessels. The renal vein should be scrutinised for evidence of tumour thrombus; if present, it is critical to assess whether it is completely resected.

- Each nodule away from the main mass (in multifocal tumours).

- Tumour-kidney interface.

- Tumour-kidney capsule.

- Areas of the capsule that are suspected of being invaded by the tumour.

- Areas of perirenal fat suspected for tumour infiltration (important for assessment whether the tumour is completely resected).

- Areas of adhesions of the tumour to surrounding tissues.

- At least two blocks of the normal kidney, and blocks from abnormal looking areas in the remaining renal tissue.

a criterion for stage III-fibrosis may be induced by preoperative chemotherapy, but in order to declare that there is non-viable tumour at resection margins, one should see either "ghost" tumour elements or foamy macrophages (figure 5A,D).

- There is clinical information on the form stating that there was a prechemotherapy tumour rupture, but there is no evidence of that at nephrectomy. The tumour should be staged objectively, according to pathological findings at nephrectomy; it is
Figure 4 Stage I tumours. (A) Viable tumour at the same level as perirenal fat, suggesting the tumour was extra-renal, but now covered by a pseudocapsule, hence, still stage I. (B) Non-viable tumour thrombus in the renal sinus vessel. (C) Chemotherapy-induced changes in the renal sinus (note a large nerve, confirming it is the sinus). (D) Chemotherapy-induced changes in the perirenal fat are not regarded as a criterion for stage II.
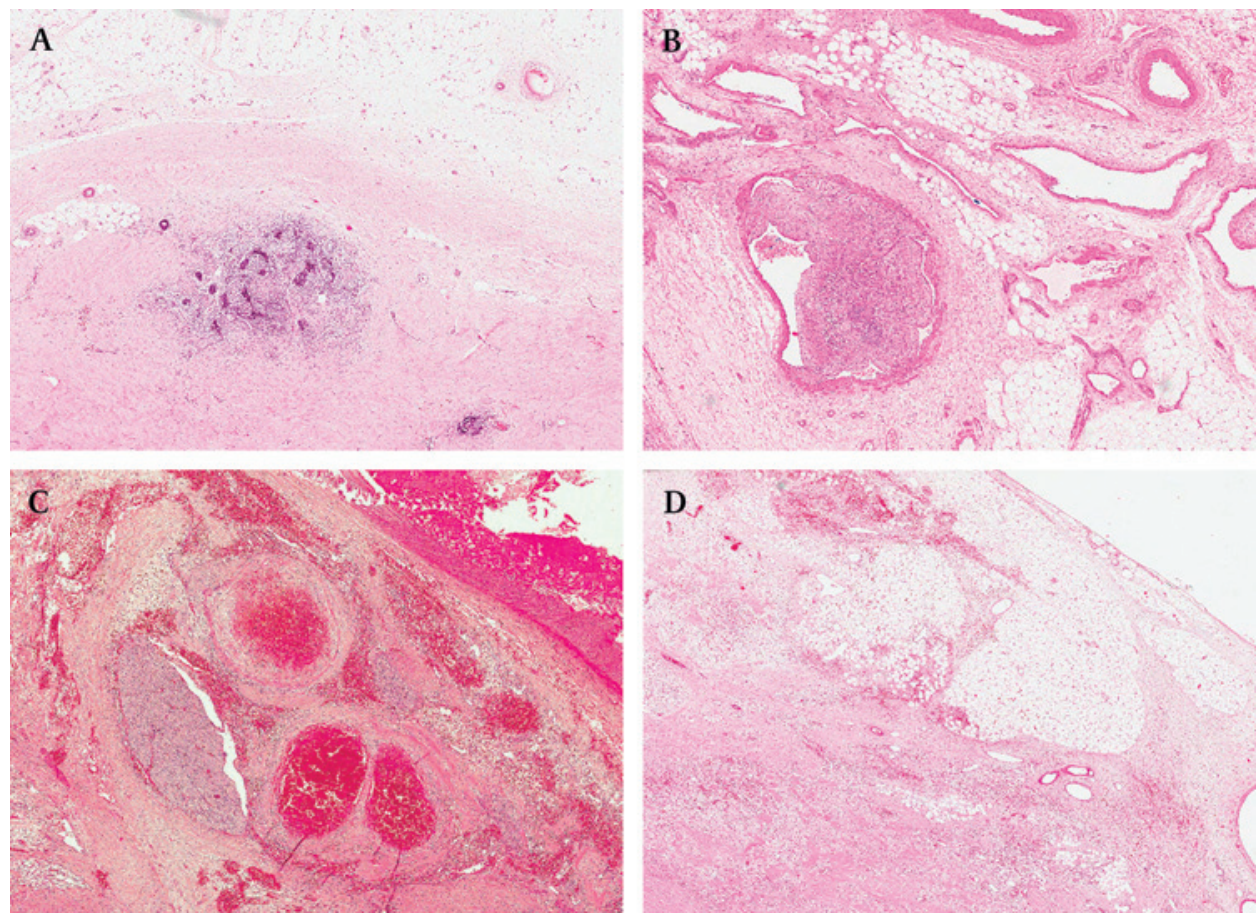
Table 3 SIOP WT 2001 staging criteria for renal tumours of childhood

\section{Stage I}

- The tumour is limited to kidney or surrounded with a fibrous (pseudo)capsule if outside of the normal contours of the kidney. The renal capsule or pseudocapsule may be infiltrated by the tumour but it does not reach the outer surface.

- The tumour may be protruding ("bulging") into the pelvic system and "dipping" into the ureter but it is not infiltrating their walls.

- The vessels or the soft tissues of the renal sinus are not involved.

- Intrarenal vessel involvement may be present.

Notes:

Fine needle aspiration or percutaneous core needle ("tru-cut") biopsy does not upstage the tumour but the size of the needle gauge should be mentioned to the pathologist.

The presence of necrotic tumour or chemotherapy-induced change in the renal sinus and/or within the perirenal fat should not be regarded as a reason for upstaging a tumour providing it is completely excised and does not reach the resection margins.

\section{Stage II}

- a) Viable tumour penetrates through the renal capsule and/or fibrous pseudocapsule into perirenal fat but is completely resected (resection margins "clear").

b) Viable tumour infiltrates the soft tissues of the renal sinus.

- c) Viable tumour infiltrates blood and lymphatic vessels of the renal sinus or in the perirenal tissue but it is completely resected.

d) Viable tumour infiltrates the renal pelvic or ureter's wall.

- e) Viable tumour infiltrates adjacent organs or vena cava but is completely resected.

\section{Stage III}

- Viable or non-viable tumour extends beyond resection margins.

- Any abdominal lymph nodes are involved.

- Tumour rupture before or intraoperatively (irrespective of other criteria for staging).

- The tumour has penetrated through the peritoneal surface.

- Tumour implants are found on the peritoneal surface.

- The tumour thrombi present at resection margins of vessels or ureter, are trans-sected or removed piecemeal by surgeon.

- The tumour has been surgically biopsied (wedge biopsy) prior to preoperative chemotherapy or surgery.

Note: The presence of necrotic tumour or chemotherapy-induced changes in a lymph node or at the resection margins is regarded as proof of previous tumour with microscopic residue and therefore the tumour is assigned stage III (because of the possibility that some viable tumour is left behind in the adjacent lymph node or beyond resection margins).

\section{Stage IV}

Haematogenous metastases (lung, liver, bone, brain, etc) or lymph node metastases outside the abdomino-pelvic region.

\section{Stage V}

Bilateral renal tumours at diagnosis. Each side should be sub-staged according to the above criteria.

the treating paediatric oncologist's decision to assign a treatment stage which may not be based on pathological findings alone.

Figure 5 Stage III tumours. (A) Chemotherapy-induced changes at the inked resection margin. (B) Non-viable tumour thrombus at the resection margin of the renal vein. (C) A part of a lymph node is replaced with non-viable tumour. (D) Non-viable blastema at the inked resection margin.
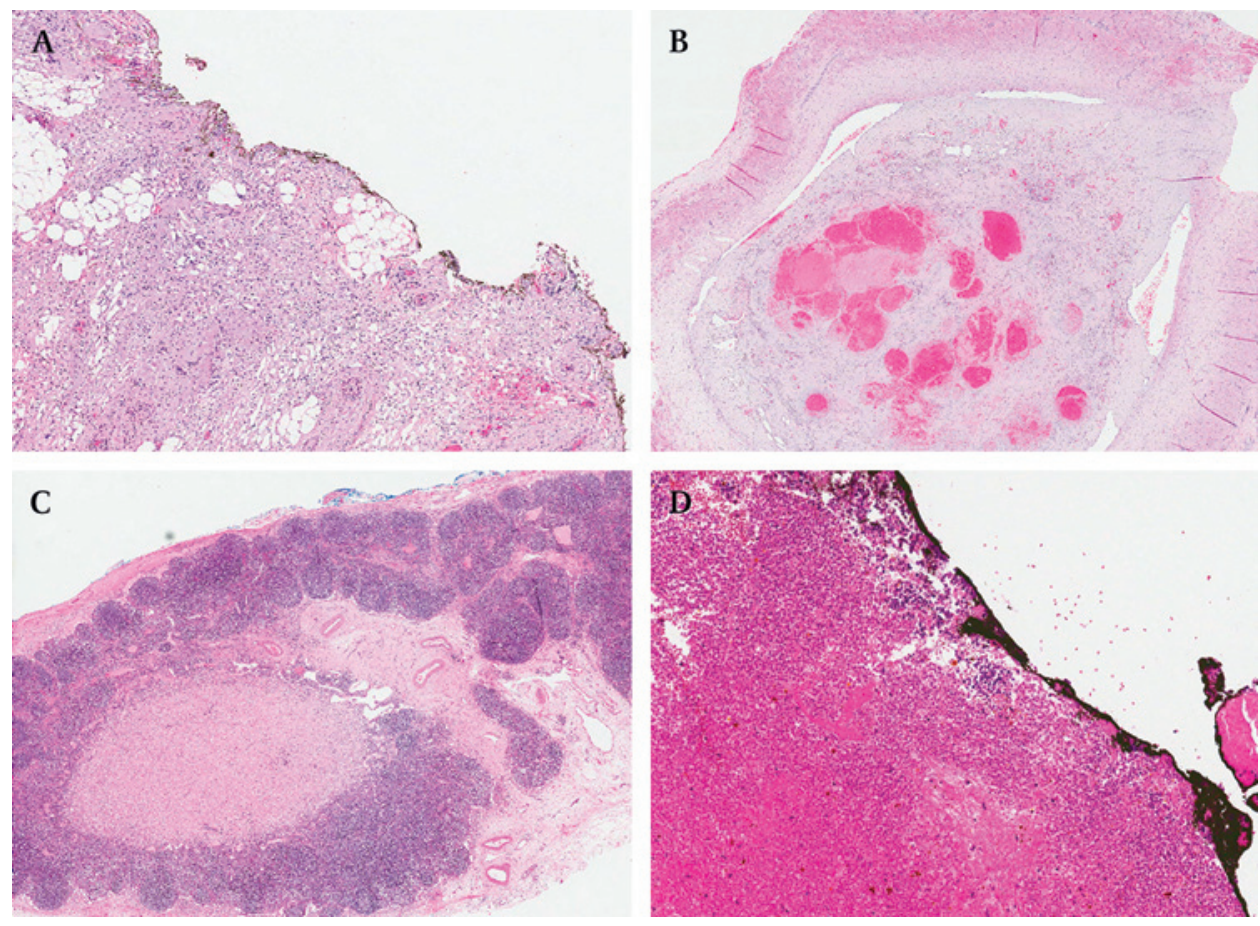
Figure 6 Viable tumour is always taken into account for staging. (A) Stage II: viable tumour thrombus in a renal sinus vessel. (B) Stage II: viable stromal thrombus showing similar features as the main tumour. (C) Stage III: viable tumour present at the inked resection margins. (D) Stage III: viable lymph node metastasis.
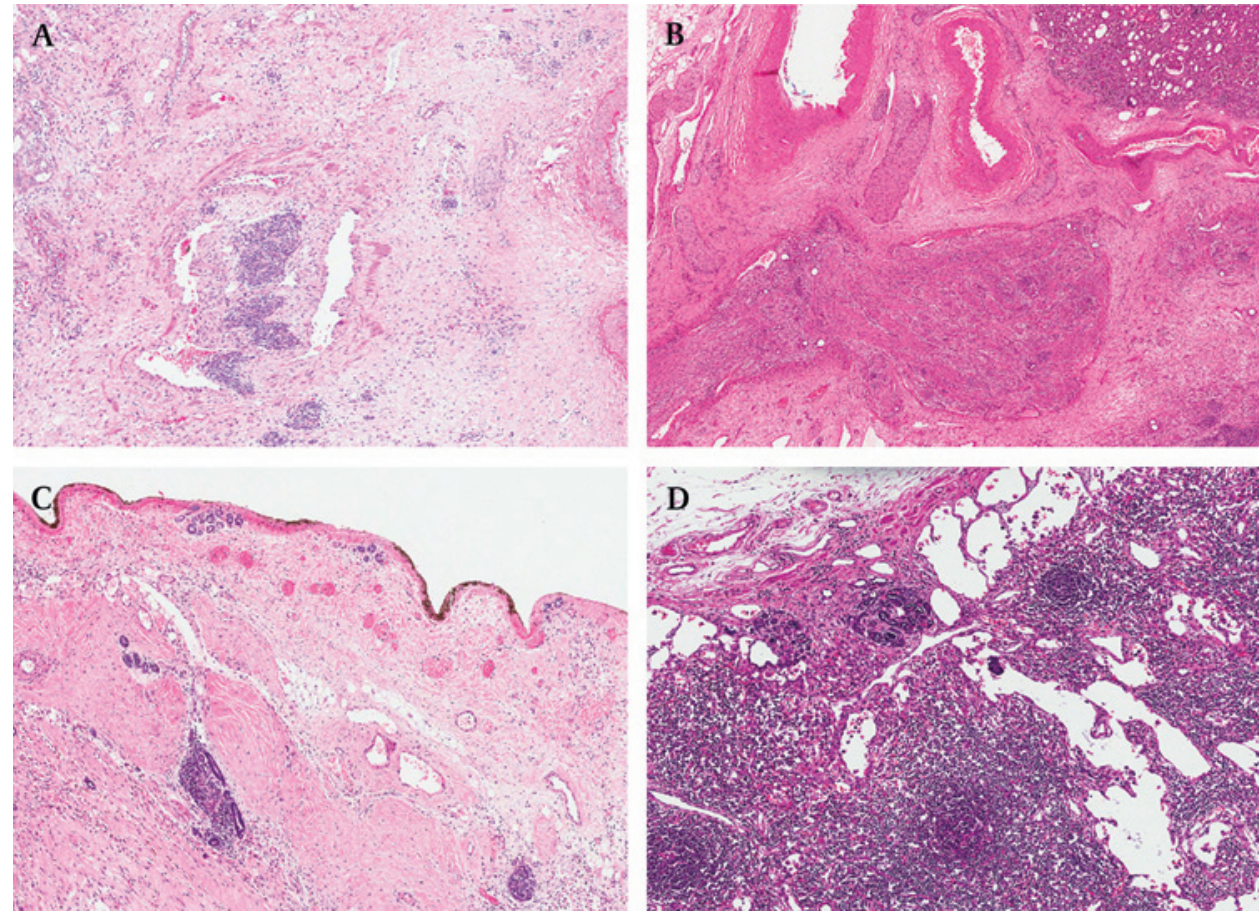

no blastema, tubules, rhabdomyoblasts or smooth muscle differentiation are present, one should compare the thrombi with the stromal component of the tumour-if identical, they should be regarded as viable (figure 6).

- What changes in a lymph node should be regarded as non-viable metastases? Only if part of a lymph node is replaced with nonviable tumour (usually in a form of foamy macrophages), should it be regarded as evidence of pre-existing tumour and staged as stage III. Massive accumulation of foamy macrophages in the peripheral sinus or within the node, without destruction of normal architecture, is not uncommon but should not upstage a tumour.

Finally, the pathology report should be crystal clear in its conclusions, which have to include tumour type, risk group, stage and reason for the stage. For example, a stage III Wilms' tumour should be reported as follows: "Wilms' tumour, regressive type, intermediate risk tumour, stage III (due to lymph node metastases-non-viable tumour)". A stage II Wilms' tumour should be reported as follows: "Wilms' tumour, mixed type, intermediate risk tumour, stage II (due to renal sinus infiltration-viable tumour)".

\section{Box 3 UK SIOP WT 2001 trial—standard operating} procedure for central pathology review

- All renal tumours should be submitted for rapid central pathology review with no delay.

- Submitted cases should include a full set of H\&E slides, (photo) block guide and the institutional pathologist's report (provisional or final).

- A set of slides for central pathology review should be prepared at the same time as the set for the institutional pathologist.

- The panel/central pathology reviewer will inform the institutional pathologist about their opinion within 24-48 hours of admission of the material.
Data from previous trials show that in around $20 \%$ of renal tumours there were diagnostic and staging discrepancies between the institutional pathologists and central pathology review. ${ }^{2}$ The current policy for the UK SIOP WT 2001 trial is that all renal tumours should be submitted for rapid central pathology review, which may allow for changes in management if necessary (box 3). ${ }^{19}$ The Royal College of Pathologists, in its recently updated guidelines, supports this view, stating that "diagnostic material from patients in a trial in which central review of the specimen is mandated in the trial protocol should be dispatched on request. If this is not done, the referring hospital will be acting against the wishes of the patient who will have given informed consent at entry into the trial, including consent for the stipulated handling of their specimens". 20

\section{Take-home messages}

- Accurate diagnosis, subtyping and staging of nephroblastoma is critical for its correct treatment; it can be achieved through a close collaboration of the institutional pathologists and a rapid central pathology review.

- Preoperative chemotherapy alters histological and staging features, and a diagnostic pathologist has to be familiar with these when dealing with the tumour.

- The assessment of chemotherapy-induced changes and viable tumour components is the basis of histological subtyping.

- The assessment is possible only if the tumour is adequately sampled.

- Non-viable tumour present in the renal sinus (both soft tissues and vessels) and perirenal fat does not upstage a tumour from stage I to stage II.

- Non-viable tumour present at the resection margins and in a lymph node upstages a tumour in stage III.

- The correct staging is only possible if the specimen is inked and there is a block guide. 
Acknowledgements The authors thank the many institutional pathologists who have over the years provided the cases on which the SIOP trials and studies are based, as well as many surgeons, paediatricians, radiation oncologists and other health professionals who managed the children entered on the SIOP trials and studies. We also thank Drs E Lazda, D Brasanac and S Popov for their critical comments on the manuscript.

Competing interests None.

Provenance and peer review Commissioned; externally peer reviewed.

\section{REFERENCES}

1. Birch JM, Breslow N. Epidemiologic features of Wilms tumor. Hematol Oncol Clin North Am 1995:9:1157-78.

2. Vujanic GM, Sandstedt B, Kesley A, et al. Central pathology review in multicentre trials and studies: lessons from the nephroblastoma trials. Cancer 2009:115:1977-83.

3. Neville HL, Ritchey ML. Wilms tumor. Overview of National Wilms Tumor Study Group results. Urol Clin North Am 2000;27:435-42.

8. de Kraker J, Graf N, van Tinteren $\mathrm{H}$, et al. Reduction of postoperative chemotherapy in children with stage I intermediate-risk and anaplastic Wilms' tumour (SIOP 93-01 trial): a randomised controlled trail. Lancet 2004;364:1229-35.

5. Beckwith JB, Palmer NF. Histopathology and prognosis of Wilms tumor. Results from the First National Wilms' Tumor Study. Cancer 1978;41:1937-48.

6. Delemarre JFM, Sandstedt B, Gerard-Marchant R, et al. SIOP Nephroblastoma trials and studies, morphological aspects. Excerpta Medica. Amsterdam 1982:261-72.

7. Vujanic GM, Delemarre JFM, Sandstedt B, et al. The new SIOP (Stockholm) working classification of renal tumours of childhood. Med Pediatr Oncol 1996:26:145-6.

8. Vujanic GM, Sandstedt B, Harms D, et al. Revised International Society of Paediatric Oncology (SIOP) working classification of renal tumors of childhood. Med Pediatr Oncol 2002;38:79-82.

9. Vujanic GM, Sandstedt B, Harms D, et al. Nephroblastoma with fibroadenomatous structures revisited. Med Pediatr Oncol 1999;32:433-5.
10. Boccon-Gibod L, Rey A, Sandstedt B, et al. Complete necrosis induced by preoperative chemotherapy in Wilms' tumor as an indicator of low risk: report of the International Society of Paediatric Oncology (SIOP) Wilms' tumor trial and study 9. Med Pediatr Oncol 2000:34:183-90.

11. Verschuur A, Vujanic G, Bohoslavsky R, et al. Wilms tumour of stromal or epithelia subtype have a good outcome in SIOP 9301 [abstract]. Pediatr Blood Cancer 2007;49:421

12. Vujanic GM, Leuschner I, Vokuhl C, et al. Subtotal chemotherapy-induced necrosis in localized non-anaplastic Wilms' tumours is an indicator of excellent prognosis-the SIOP 9301 results [abstract]. Book of abstracts of the 54th Paediatric Pathology Society annual meeting, Helsinki, 4-6 September 2008:4.

13. Weirich A, Leuschner I, Harms D, et al. Clinical impact of histologic subtypes in localized non-anaplastic nephroblastoma treated according to the trial and study SIOP-9/GPOH. Ann Oncol 2001;12:311-9.

14. Mitchell C, Pritchard Jones K, Shannon R, et al. Immediate nephrectomy versus preoperative chemotherapy in the management of non-metastatic Wilms' tumor: results of a randomized trial (UKWT3) by the UK Children's Cancer Study Group. Eur J Cancer 2006:42:2554-62.

15. Vujanic GM, Kelsey A, Mitchell C, et al. The role of biopsy in the diagnosis of renal tumors of childhood: results of the UKCCSG Wilms Tumor Study 3. Med Pediatr Oncol 2003:40:18-22.

16. Dome JS, Cotton CA, Perlman EJ, et al. Treatment of anaplastic Wilms' tumor: results from the fifth National Wilms' Tumor Study. J Clin Oncol 2006:24:2352-8.

17. Faria P, Beckwith JB, Mishra K, et al. Focal versus diffuse anaplasia in Wilms tumor-new definitions with prognostic significance. A report from the National Wilms Tumor Study Group. Am J Surg Pathol 1996;20:909-20.

18. Vujanić GM, Harms D, Bohoslavsky R, et al. Non-viable tumour tissue should not upstage Wilms' tumour from stage I to stage II-a report from the SIOP 93-01 nephroblastoma trial and study. Pediatr Develop Pathol 2009;12:111-5.

19. SIOP WT 2001 Clinical Trial and Study Protocol, 2001.

20. Furness $\mathbf{P}$. Guidelines on inter-departmental dispatch of samples from patients sent to another hospital or centre for assessment and/or treatment, 2nd edn. London: The Royal College of Pathologists, 2006. 